\title{
Receding horizon control of mixed line flow shop systems
}

\author{
Kagan Gokbayrak
}

Received: 17 November 2009 / Accepted: 29 October 2010 / Published online: 12 November 2010

(C) Springer Science+Business Media, LLC 2010

\begin{abstract}
We consider reliable mixed line flow shop systems that are composed of controllable and uncontrollable machines. These systems are assumed to receive arrivals at random instants and process jobs deterministically in the order of arrival so as to depart them before their deadlines that are revealed at the time of arrival. We model these flow shops as serial networks of queues operating under a nonpreemptive first-come-first-served policy. Defining completion-time costs for jobs and process costs at controllable machines, a stochastic convex optimization problem is formulated where the control variables are the constrained service times of jobs at the controllable machines. As an on-line solution method to determine these service times, we propose a receding horizon controller, which solves a deterministic problem at each decision instant. We quantify the available future information by the look-ahead window size. Numerical examples demonstrate the value of information and that the no-waiting property of the full-information case is not observed in the partial-information case.
\end{abstract}

Keywords Manufacturing • Queueing systems • Receding horizon control • Controllable service times $\cdot$ Value of information

\section{Introduction}

We consider reliable manufacturing flow shop systems composed of CNC (Computer Numerical Control) and conventional machines. Job arrivals occur at random times with possibly nonstationary interarrival time distributions. The service times at machines, however, are deterministic, and jobs are processed in the order they arrive; therefore, we model these systems as serial networks of G/D/1 queues

K. Gokbayrak $(\bowtie)$

Department of Industrial Engineering, Bilkent University, Ankara, 06800, Turkey

e-mail:kgokbayr@bilkent.edu.tr 
with FIFO (first-in-first-out) discipline. Assuming that conventional machines are uncontrollable with fixed service times, our objective is to determine the service times at the $\mathrm{CNC}$ machines so as to depart jobs before their deadlines, which are revealed at the times of their arrivals.

While determining the service times at the $\mathrm{CNC}$ machines, in addition to satisfying the hard deadline constraints, we want to minimize a total cost of production formed of process and completion-time costs. Selecting smaller service times, which corresponds to faster processing, incurs higher tooling costs due to wear and tear caused by increased surface temperatures. The quality losses due to faster processing are also lumped into the process costs along with the tooling costs. Therefore, we assume that the process costs are decreasing in the service times. The completion-time costs, on the other hand, are the work-in-process inventory costs that are assumed to be increasing in the completion times. Note that, even though the completiontimes are upper bounded by deadlines that are promised to the customers, different completion-times within these bounds occur different costs for the system; therefore, the completion-time costs are also included in the objective. Since longer services may delay completions increasing completion-time costs while decreasing process costs, a trade-off is observed in the service time selection. A stochastic optimal control problem defined over a max-plus algebra is formulated in the next section demonstrating this trade-off.

The work in Gazarik and Wardi (1998) started the literature on optimal control of discrete-event dynamic processes defined over a max-plus algebra. In that work, a single machine processing a given sequence of jobs is considered. The problem in question is how to optimally control the completion times of the jobs by assigning their release (arrival) times, so as to minimize a measure of the discrepancy between the completion times and given desired due dates. In Pepyne and Cassandras (2000), Pepyne and Cassandras considered the same single machine system with a similar problem where instead of assigning release times, service times were assigned. The objective function was designed to complete jobs with the minimal effort penalizing deviations from given due dates. The uniqueness of the optimal solution for this problem was shown in Cassandras et al. (2001). Exploiting the structural properties of the optimal sample path, efficient solution algorithms to obtain this unique solution were developed in Wardi et al. (2001), Cho et al. (2001), and Zhang and Cassandras (2002). For the case where job arrival times are random, in Cassandras and Mookherjee (2003a, b, c), Cassandras and Mookherjee proposed a receding horizon controller employing the forward decomposition algorithm developed in Cho et al. (2001) and analyzed the effect of the look-ahead window size on the optimal costs. Adding job completion deadlines as hard constraints and removing the completion time costs, Mao et al., in Mao et al. (2007), considered an optimization problem for a single machine system based only on process costs. Under the assumption that these costs are decreasing convex, they showed that the optimal solution characteristics lead to the highly efficient Critical Task Decomposition Algorithm (CTDA). Employing CTDA, Miao and Cassandras, in Miao and Cassandras (2007), developed a receding horizon controller for this system for the case where the job arrival times and deadlines are random. Analyzing sample paths obtained under this controller and comparing them to optimal sample paths (obtained when all event times are known), they derived a number of properties of the controller. Unfortunately, the receding horizon controllers developed for single machine systems cannot be directly applied to flow shop systems due to interactions between machines. 
The optimal control of flow shop systems with regular costs on completion times and decreasing costs on service times was considered in Gokbayrak and Selvi (2007) by Gokbayrak and Selvi for the case where all machines are controllable and all arrival times are initially known. Under strict convexity assumptions for costs, it was shown that, on the optimal path, jobs do not wait between machines, a property that enables the transformation of the non-smooth discrete-event optimal control problem into a simple convex programming problem. An efficient forward-in-time decomposition algorithm was also proposed for its solution. In Gokbayrak and Selvi (2008, 2009), Gokbayrak and Selvi proved waiting characteristics in flow shop systems composed of only fixed-service-time machines. Building on the findings from these three papers, Gokbayrak and Selvi generalized the no-wait property to mixed line flow shop systems formed of controllable and uncontrollable machines operating under the cost structure in Gokbayrak and Selvi (2007) with the additional hard deadline constraints: no waiting is observed downstream to the first controllable machine. The generalized version of the forward decomposition algorithm was also proposed in Gokbayrak and Selvi (2010).

In this paper, we consider the optimization problem in Gokbayrak and Selvi (2010), with the modification that arrival time and deadline information are only available for jobs that already arrived or are about to arrive within a look-ahead window. As an on-line solution method for the resulting stochastic optimal control problem, we propose a receding horizon controller. The amount of available future information is quantified by the look-ahead window size. We show, by numerical examples, that the sample path costs decrease with increasing window sizes down to the cost of the full-information case, the case where all arrival times and deadlines are initially available.

The rest of the paper is organized as follows: In Section 2, we formulate the stochastic optimization problem under consideration, which is defined over a nonconvex domain. Under the same cost assumptions as in Gokbayrak and Selvi (2010), an equivalent stochastic convex optimization problem is formulated and the receding horizon controller is proposed for its solution. Section 3 demonstrates the effect of look-ahead window size, and presents a counter-example for the no-waiting property. Finally, in Section 4, the conclusion is presented.

\section{Problem formulation}

Let us consider an $M$-machine flow shop system consisting of controllable and uncontrollable machines. The machines are indexed from the set $\{1, \ldots, M\}$ according to their location in the flow shop. A sequence of $N$ jobs arrive at random times $\left\{a_{i}\right\}_{i=1}^{N}$ and are indexed so that $0 \leq a_{1} \leq a_{2} \leq \ldots \leq a_{N}$. These jobs, which we denote by $\left\{C_{i}\right\}_{i=1}^{N}$, are processed at all machines sequentially on a first-come first-served nonpreemptive basis. There are completion deadlines, represented by $\left\{d_{i}\right\}_{i=1}^{N}$, that are revealed at each job's arrival time. In the following, we denote the departure time of job $C_{i}$ from machine $j$ by $x_{i, j}$, and the completion-time cost for this job is represented by $\phi_{i}\left(x_{i, M}\right)$.

We define the sets $I_{C}$ and $I_{U}$, both subsets of the set $I=\{1, \ldots, M\}$, as the index sets of the controllable and the uncontrollable machines, respectively. The durations of processes at each machine $j$ are denoted by the service times $\left\{s_{i, j}\right\}_{i=1}^{N}$. Due to 
physical limitations of the machines, we assume that each job at a controllable machine $j \in I_{C}$ needs at least a service of $S_{j}$ duration. There is no upper bound on the service times at the controllable machines. A process cost $\theta_{j}\left(s_{i, j}\right)$ is associated with each job served at a controllable machine $j$ for a duration of $s_{i, j}$. We assume that the service times at the uncontrollable machines $j \in I_{U}$ are fixed to values $s_{j}$ for all jobs.

We consider the discrete-event stochastic optimal control problem, denoted by $P$, which has the following form:

$$
P: \min \left\{J=E\left[\sum_{i=1}^{N} \sum_{j \in I_{C}} \theta_{j}\left(s_{i, j}\right)+\sum_{i=1}^{N} \phi_{i}\left(x_{i, M}\right)\right]\right\}
$$

subject to

$$
\begin{array}{rlrl}
x_{i, j} & =\max \left(x_{i, j-1}, x_{i-1, j}\right) & & s_{i, j} \quad \forall j \in I \\
x_{i, M} & \leq d_{i} & & \\
s_{i, j} & =s_{j} & \forall j \in I_{U} \\
s_{i, j} \geq S_{j} & \forall j \in I_{C} \\
x_{i, 0} & =a_{i}, \quad x_{0, j}=-\infty & & \forall j \in I
\end{array}
$$

for all $i=1, \ldots, N$.

Note that since the arrival times and the deadlines are random, we define an expected total cost $J$. The Lindley-type equation (2) and its boundary conditions (6) define the departure time dynamics of jobs in a serial queueing network. Job deadlines and physical constraints on service times at the controllable machines are given in Eqs. 3 and 5, respectively. The service times at the uncontrollable machines are set to fixed values as in Eq. 4.

As in Gokbayrak and Selvi (2010), we assume that the first machine of the flow shop is controllable. For flow shop systems starting out with a number of uncontrollable machines, we can reformulate the problem so that the uncontrollable portion at the beginning is dropped. Since the service times at these machines are fixed, this modification has no effect on the optimal service times at the controllable machines. Note that the arrival times also have to be redefined via Eqs. 2 and 6 to reflect the arrival times at the first controllable machine.

Since the service times are either fixed or lower-bounded, it may not be possible to satisfy the deadline constraints in Eq. 3. In this work, we assume that a feasible solution exists for $P$. If not, a mixed integer programming problem can be formulated as in Mao and Cassandras (2010) to reject some of the jobs for feasibility. The job admission problem is a subject of ongoing research, and it is not considered here.

Following upon the discussion in the previous section, two standing assumptions are stated to make the problem somewhat more tractable while preserving the originality of the problem.

Assumption $1 \theta_{j}(\cdot)$, for $j \in I_{C}$, is continuously differentiable, monotonically decreasing, and strictly convex. 
Assumption $2 \phi_{i}(\cdot)$, for $i=1, \ldots, N$, is continuously differentiable, monotonically increasing, and convex.

Due to the $\max$ functions in the constraints, $P$ is defined over a non-convex domain. Linearizing these constraints in Eq. 2 and employing the boundary constraints in Eq. 6, we can formulate a stochastic convex optimization problem:

$$
\bar{P}: \min \left\{J=E\left[\sum_{i=1}^{N} \sum_{j \in I_{C}} \theta_{j}\left(s_{i, j}\right)+\sum_{i=1}^{N} \phi_{i}\left(x_{i, M}\right)\right]\right\}
$$

subject to

$$
\begin{array}{rlrl}
x_{i, 1} & \geq a_{i}+s_{i, 1} & & \forall i \\
x_{i, j} \geq x_{i, j-1}+s_{i, j} & & \forall i, \forall j>1 \\
x_{i, j} \geq x_{i-1, j}+s_{i, j} & & \forall i>1, \forall j \\
x_{i, M} & \leq d_{i} & & \forall i \\
s_{i, j} & =s_{j} & & \forall i, \forall j \in I_{U} \\
s_{i, j} & \geq S_{j} & & \forall i, \forall j \in I_{C}
\end{array}
$$

Note that the feasible region for $\bar{P}$ includes the feasible region for $P$. Therefore, one can argue that $\bar{P}$ can have an optimal solution outside the feasible region of $P$. Due to the standing assumptions, however, it can be shown as in Gokbayrak and Selvi (2007) that the optimal solution of $\bar{P}$ resides in the feasible set of $P$ and it is also the optimal solution of $P$. (The idea behind this claim is that if the optimal solution of $\bar{P}$ were not in the feasible region of $P$, then for some $i$ and $j$ we would have none of the constraints (7-9) active. In that case, we could decrease $x_{i, j}$ further until one of these constraints became active. By Assumption 2, this modified solution would have a lower cost than the optimal solution resulting with a contradiction.) Hence, we can concentrate on solving $\bar{P}$ instead.

Since the arrival times and the deadlines are random with possibly nonstationary and unknown distributions, in order to determine the service times we resort to receding horizon techniques described next.

\subsection{Receding horizon control}

Receding horizon controllers operate on-line with the available information as if no other job is expected to arrive. Every time a service time decision needs to be made, receding horizon controllers solve deterministic optimization problems for jobs that are either already observed or to be observed within a look-ahead window of size $\omega$. Since previous decisions, which are already applied, cannot be changed, they have to be incorporated in the optimization problem as constraints. 
At a decision time $t$ when a job is about to start service at some controllable machine, we have the arrival and deadline information available for jobs $\left\{C_{i}\right\}_{i=1}^{n}$ where $n$ satisfies

$$
a_{n} \leq t+\omega<a_{n+1}
$$

Let us represent the lowest index job in the system by $C_{k}$. Then, the following deterministic problem is solved to determine the service times:

$$
Q\left(k, n, D_{t}\right): \min \left\{\sum_{i=k}^{n} \sum_{j \in I_{C}} \theta_{j}\left(s_{i, j}\right)+\sum_{i=k}^{n} \phi_{i}\left(x_{i, M}\right)\right\}
$$

subject to

$$
\begin{array}{rlrl}
x_{i, 1} \geq a_{i}+s_{i, 1} & & \forall i \\
x_{i, j} \geq x_{i, j-1}+s_{i, j} & & \forall i, \forall j>1 \\
x_{k, j} \geq \bar{x}_{k-1, j}+s_{k, j} & & \forall j \\
x_{i, j} \geq x_{i-1, j}+s_{i, j} & & \forall i>k, \forall j \\
x_{i, M} \leq d_{i} & & \forall i \\
s_{i, j} & =s_{j} & & \forall i, \forall j \in I_{U} \\
s_{i, j} & \geq S_{j} & & \forall i, \forall j \in I_{C} \\
s_{i, j} & =\bar{s}_{i, j} & & \forall(i, j) \in D_{t}
\end{array}
$$

where $D_{t}$ is the set of job and controllable machine index pairs for service times that are previously decided and already applied by time $t$ (i.e., these services have started with service times $\bar{s}_{i, j}$ ) and $\bar{x}_{k-1, j}$ is the realized departure time of job $C_{k-1}$ from machine $j$. In the case of $k=1$, we assume $\bar{x}_{k-1, j}=0$ for all $j$.

In the random arrivals and deadlines setting, we quantify the amount of future information by the look-ahead window size $\omega$. From Eq. 13, we conclude that a larger window size $\omega$ may increase the index $n_{r}$. As a result, the effect of additional future jobs can also be taken into account. Next, we show by numerical examples that more future information improves the service time decisions lowering the sample path costs.

\section{Numerical examples}

In this section, we present two numerical examples to illustrate the value of future information and that no waiting property of the full-information case may not hold when receding horizon controllers are employed. 
Example 1 We consider the example from Gokbayrak and Selvi (2007) where the number of machines is $M=4$ and the number of jobs is $N=10$. Service and completion-time costs are selected to be

$$
\theta_{j}\left(s_{i, j}\right)=\frac{\beta_{j}}{s_{i, j}}
$$

and

$$
\phi_{i}\left(x_{i, M}\right)=\alpha\left(x_{i, M}-a_{i}\right)^{2}
$$

respectively, where $\beta=[10,5,20,10]$ and $\alpha=10$. Job completion deadlines and lower bounds on service times are selected such that the corresponding constraints are never active.

We analyze the effect of a look-ahead window size $\omega$ on a single sample path, where the arrival times are $a=[0.0,2.3,2.4,4.9,5.0,5.5,9.0,9.5,11.0,13.0]$. From Gokbayrak and Selvi (2007), the optimal cost, when all arrival times are initially available (full information case, $\omega=\infty$ ), is known to be 1290.15 . The effect of window size on cost is illustrated in Fig. 1. For $\omega=0$, the cost comes out as 1308.9. (Small cost differences are typical when the full information optimal sample path has busy periods formed of small numbers of jobs). Note that for $\omega=0.6$, we already have enough information to obtain the full-information optimal solution.

As expected, the solution cost is a non-increasing function of the window size: For a range of window sizes, the same sequence of problems are solved. We see drops in the cost value only when an additional job is introduced to one of these problems by an increase in the $\omega$ value. Hence, for each sample path, a descending step behavior is observed.

If one observes several sample paths, the ensemble average should come out as a smoother decreasing function, which is illustrated in the next example.

Fig. 1 Value of information

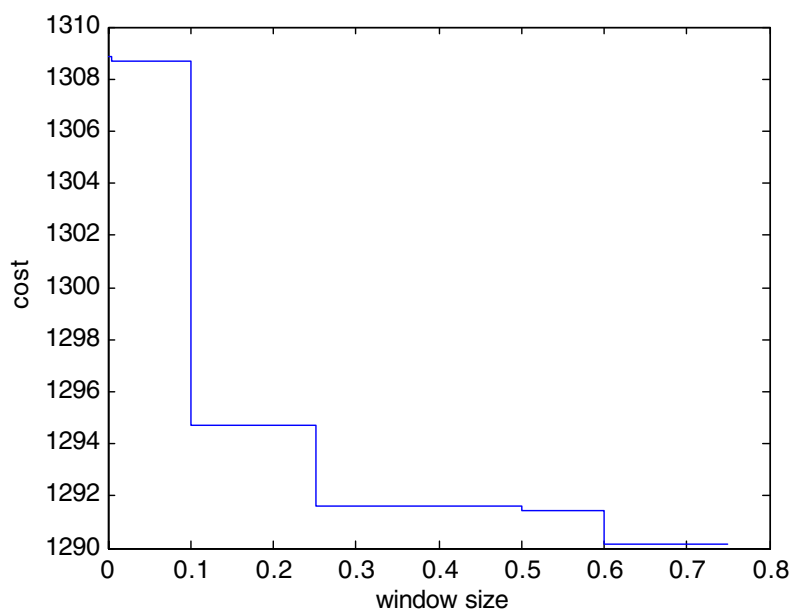


Fig. 2 Ensemble average cost

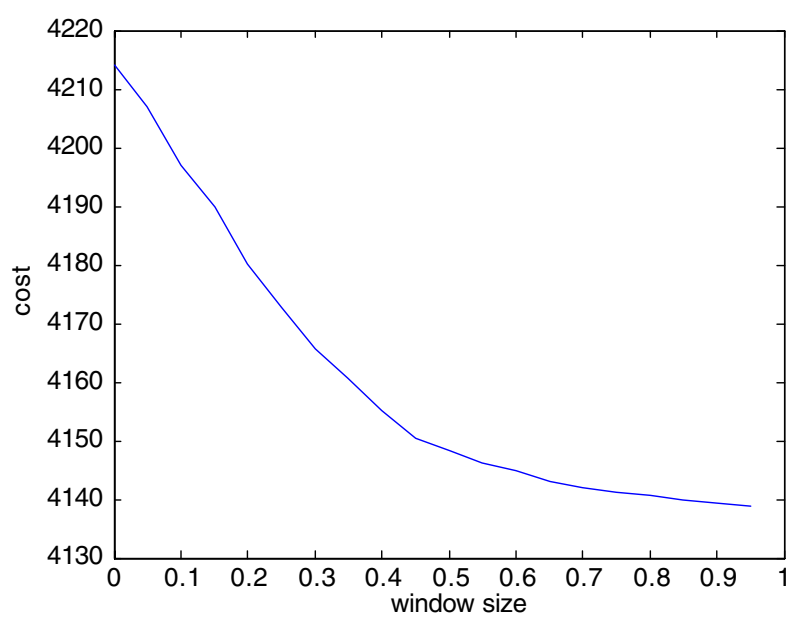

Example 2 We modify the first example to a five-machine flow shop system with $\beta=[10,5,20,10,30]$ and $\alpha=10$, processing 20 jobs arriving according to a Poisson process with rate 1 . Fifty sample paths are observed and the ensemble averages are plotted in Fig. 2 for various window sizes to illustrate the value of information.

Note that the incremental value of information decreases by the window size, i.e., for small $\omega$ values, additional information has more expected value.

In Gokbayrak and Selvi (2010), it was shown that no waiting is observed after the first controllable machine. Receding horizon controllers, however, solve a similar problem with additional previous decision constraints. The next example illustrates that waiting is possible due to these additional constraints.

Example 3 Let us modify the first example to have only three jobs with arrivals at $a=[0,1,1]$ and two controllable machines with $\beta=[10,10000]$. The resulting system size (the total number of jobs in the queue and in the server) for the second machine is shown in Fig. 3: The receding horizon controller forces the second job

Fig. 3 System size at the second machine

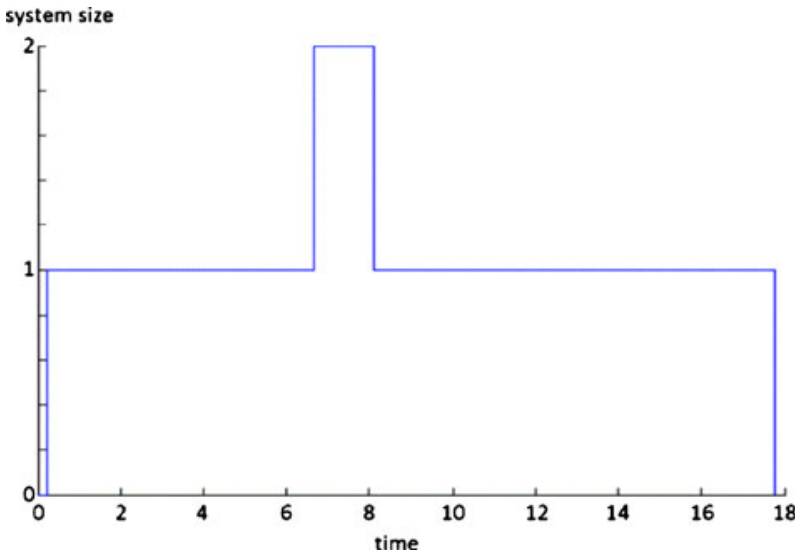


to wait for service at the second machine, so the 'no-waiting' property of the fullinformation case does not hold for the problems solved by the receding horizon controllers.

\section{Conclusion}

We considered a stochastic version of the optimization problem in Gokbayrak and Selvi (2010) for flow shop systems composed of both controllable and uncontrollable machines. In the absence of a priori knowledge of arrival times and job deadlines, we proposed a receding horizon controller that solves deterministic optimization problems formulated with the information available at each decision instant.

The value of future information was shown by two numerical examples. The lack of information causes the controller to be myopic. Previous decisions, which may not be optimal for the full information problem, result with additional constraints for the optimization problems, possibly removing the full information solution from the feasible set and therefore increasing the costs. We also showed by a counter-example that the 'no-waiting' property for the full information case does not necessarily hold for the partial information case, due to these previous decision constraints.

\section{References}

Cassandras CG, Mookherjee R (2003a) Receding horizon control for a class of hybrid systems with event uncertainties. In: Proc. of 2003 American control conf, pp 5197-5202

Cassandras CG, Mookherjee R (2003b) Properties of receding horizon controllers for some hybrid systems with event uncertainties. In: Proc. 2003 IFAC conf. on analysis and design of hybrid systems, pp 413-418

Cassandras CG, Mookherjee R (2003c) Receding horizon optimal control for some stochastic hybrid systems. In: Proc. of 42nd IEEE conf. decision and control, pp 2162-2167

Cassandras CG, Pepyne DL, Wardi Y (2001) Optimal control of a class of hybrid systems. IEEE Trans Automat Contr 46(3):398-415

Cho YC, Cassandras CG, Pepyne DL (2001) Forward decomposition algorithms for optimal control of a class of hybrid systems. Int J Robust Nonlinear Control 11:497-513

Gazarik M, Wardi Y (1998) Optimal release times in a single server: an optimal control perspective. IEEE Trans Automat Contr 43(7):998-1002

Gokbayrak K, Selvi O (2007) Constrained optimal hybrid control of a flow shop system. IEEE Trans Automat Contr 52(12):2270-2281

Gokbayrak K, Selvi O (2008) Optimization of a flow shop system of initially controllable machines. IEEE Trans Automat Contr 53(11):2665-2668

Gokbayrak K, Selvi O (2009) A subgradient descent algorithm for optimization of initially controllable flow shop systems. Discret Event Dyn Syst: Theor Appl 19(2):267-282

Gokbayrak K, Selvi O (2010) Service time optimization of mixed line flow shop systems. IEEE Trans Automat Contr 55(2):395-404

Miao L, Cassandras C (2007) Receding horizon control for a class of discrete event systems with real-time constraints. IEEE Trans Automat Contr 52(5):825-839

Mao J, Cassandras CG (2010) Optimal admission control of discrete event systems with real-time constraints. Discret Event Dyn Syst 20(1):37-62

Mao J, Cassandras CG, Zhao Q (2007) Optimal dynamic voltage scaling in energy-limited nonpreemptive systems with real-time constraints. IEEE Trans Mob Comput 6-6:678-688 
Pepyne DL, Cassandras CG (2000) Optimal control of hybrid systems in manufacturing. Proc IEEE 88(7):1108-1123

Wardi Y, Cassandras CG, Pepyne DL (2001) A backward algorithm for computing optimal controls for single-stage hybrid manufacturing systems. Int J Prod Res 39(2):369-393

Zhang P, Cassandras CG (2002) An improved forward algorithm for optimal control of a class of hybrid systems. IEEE Trans Automat Contr 47(10):1735-1739

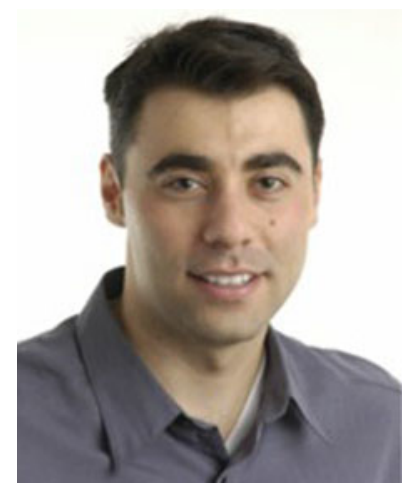

Kagan Gokbayrak was born in Istanbul, Turkey, in 1972. He received the B.S. degrees in mathematics and in electrical engineering from Bogazici University, Istanbul, the M.S. degree in electrical and computer engineering from the University of Massachusetts, Amherst, and the Ph.D. degree in manufacturing engineering from Boston University, Boston, MA, in 1995, 1995, 1997, and 2001, respectively. From 2001 to 2003, he worked as a Network Planning Engineer at Genuity, Inc., Burlington, MA. Since 2003, he has been a faculty member in the Industrial Engineering Department of Bilkent University, Ankara, Turkey. He specializes in the areas of discrete-event and hybrid systems, stochastic optimization, and computer simulation, with applications to inventory and manufacturing systems. 